\title{
Pluraler Frieden: Eine Perspektive für die OSZE?
}

\author{
Matthias Dembinski, Hans-Joachim Spanger"
}

\section{Zusammenfassung}

Die OSZE steckt in einer tiefen Krise. Statt ihre Kernaufgabe zu erfüllen und zur Zivilisierung des russisch-westlichen Konflikts beizutragen, wird sie von diesem Konflikt zerrieben. Dies hängt auch mit ihrem Design zusammen, das nicht mehr zu der Konfliktstruktur passt. Wir stellen zwei Optionen ihrer institutionellen Anpassung vor. Die erste würde das Aufgabenspektrum der OSZE auf den am ehesten konsensfähigen Bereich der Konfliktprävention beschränken und die Handlungsfähigkeit in diesem Bereich durch die Delegation von Kompetenzen an ständige Organe sichern. Die zweite würde den Dialog und die Suche nach Übereinstimmung ins Zentrum stellen, die OSZE aber gleichzeitig operativ entlasten. Dies beschränkt zwar ihre Handlungsfähigkeit, stärkt zugleich aber ihre Legitimität.

\section{Schlagworte}

pluraler Frieden; institutionelles Design; Krise der OSZE; russisch-westlicher Konflikt

Bitte zitieren als: Matthias Dembinski und Hans-Joachim Spanger, Pluraler Frieden: Eine Perspektive für die OSZE?, IFSH (Hrsg.), OSCE Insights 9/2021 (Baden-Baden: Nomos, 2022), https://doi.org/10.5771/9783748911449-09

\section{Einleitung ${ }^{1}$}

Um das, was früher als Ost-West-Beziehung bezeichnet wurde - also im Wesentlichen das Verhältnis zwischen Russland und den westlichen Staaten - steht es aktuell denkbar schlecht. Die militä-

* Dr. Matthias Dembinski

Hessische Stiftung Friedens- und Konfliktforschung dembinski@hsfk.de

Dr. Hans-Joachim Spanger

Hessische Stiftung Friedens- und Konfliktforschung spanger@hsfk.de rischen Spannungen sind seit der Annexion der Krim und dem Krieg im Osten der Ukraine gefährlich hoch, WorstCase-Szenarien und stereotype Verdächtigungen bestimmen das Denken, hybride Kriegführung ist en vogue. Ende 2021 wird sogar über eine großangelegte russische Invasion der Ukraine Anfang des Jahres 2022 spekuliert. Versuche, auf der Basis gemeinsamer Interessen Chancen der Kooperation auszuloten, werden diskreditiert. Dieses konfrontative Verhalten hat sich weitgehend von den Konfliktgegenständen entkoppelt. Obwohl beide Seiten immer wieder ihre prinzipielle Bereitschaft zur (selektiven) Kooperati- 
on bekunden, wächst die Polarisierung. Dabei erodieren die Begrenzungen zwischen Politikfeldern mit gemeinsamen und solchen mit antagonistischen Interessen, überlagern die antagonistischen Handlungsmuster die gemeinsamen Interessen und bildet sich eine umfassende Konfrontationslogik heraus.

Der Konflikt hat praktisch alle Kooperationsformate zwischen Russland und dem Westen in Mitleidenschaft gezogen. Das gilt für den NATO-Russland-Rat ebenso wie für die institutionalisierte Kooperation zwischen Russland und der EU. Als einzige umfassende Institution bleibt also die Organisation für Sicherheit und Zusammenarbeit in Europa (OSZE), der alle europäischen sowie die zentralasiatischen Länder und die Mongolei angehören. Sie wurde expressis verbis dafür geschaffen, vertrauensbildend $\mathrm{zu}$ wirken. Aufgrund ihrer Geschichte und ihrer institutionellen Ressourcen ist die OSZE prinzipiell in der Lage, Felder gegensätzlicher Interessen zu isolieren und auf Feldern gemeinsamer Interessen Kooperation zu organisieren. Allerdings wird seit 2014 auch die OSZE von der ungebremsten Konfliktdynamik zwischen Russland und dem Westen zerrieben. Statt Spannungen zu reduzieren, ist sie zum Austragungsort des Konflikts geworden.

Diese institutionelle Krise der OSZE stellt ein Puzzle dar. Zum einen wird der Vorgängerin der OSZE, der Konferenz über Sicherheit und Zusammenarbeit in Europa (KSZE), allgemein attestiert, sie habe in der antagonistischen Ost-West-Konstellation des Kalten Kriegs zur Einhegung des Konflikts und sogar zu seiner Transformation beigetragen. Zum anderen weisen die einvernehmlichen Beschlüsse etwa zur Schaffung der Special Monitoring Mission in der Ostukraine darauf hin, dass sich die OSZETeilnehmerstaaten nach wie vor bewusst sind, wie nützlich institutionelle Arrangements sind, um Konflikte einzuhegen. Es stellt sich daher die Frage, warum es diese Lücke gibt zwischen dem Bedarf und dem Interesse an institutioneller Konfliktbearbeitung und der Unwilligkeit der betroffenen Staaten, die von ihnen für diesen Zweck geschaffene Organisation zu nutzen. In diesem Beitrag diskutieren wir, ob und wie diese Lücke durch einen Umbau der OSZE geschlossen werden kann.

Wir entfalten dieses Argument, indem wir in einem ersten Schritt mit Rückgriff auf die Literatur zu Krisen institutioneller Ordnungen zeigen, dass und wie Institutionen, die von den Staaten selbst geschaffen wurden, umstritten und selbst Gegenstand von Konflikten werden können. In einem zweiten Schritt erfolgt die Anwendung auf die OSZE und ihre Vorläuferin, die KSZE. Auf der Grundlage dieser Analyse und in Anlehnung an unser Konzept eines "Pluralen Friedens" fragen wir in einem weiteren Schritt, was zu tun ist und wie sich die OSZE so umbauen ließe, damit sie wieder zum konstruktiven Konfliktmanagement beitragen kann. Dazu schlagen wir zwei Reformpfade vor: eine institutionelle Vertiefung der OSZE bei gleichzeitiger Beschränkung ihres Aufgabenspektrums und alternativ eine Rückbesinnung auf das konsensbildende Format der KSZE. 


\section{Zur Krise internationaler Institutionen}

Lange begriff die Forschung Institutionen strikt als Instrumente, die von den Staaten mit dem Ziel geschaffen werden, Kooperation zu verstetigen, Transaktionskosten zu reduzieren und so Kooperation effizienter zu gestalten. Weil Institutionen und ihr Design - die Mitgliedschaft, die thematische Reichweite, ihre Regeln und Normen sowie ihre Organe und Abstimmungsverfahren - von den Mitgliedstaaten im Konsens bestimmt werden, galten institutionelle Krisen und ein Infragestellen der Institutionen als unwahrscheinlich. Dass Institutionen von ihren Mitgliedern als fremd wahrgenommen werden, umstritten sind und sogar Spannungen verschärfen können, gerät erst allmählich und mit der Krise der internationalen liberalen Ordnung in den Blick. ${ }^{2}$ Dabei sind die Mechanismen, die erklären, warum Institutionen umstritten werden, schnell benannt.

Erstens gehen bei der Gründung einer Institution in der Regel verschiedene und oft auch gegensätzliche Vorstellungen in ihr Design ein. Zu diesem Zeitpunkt lässt sich noch nicht abschätzen, wie sich ihre Regeln und Verfahren in der Praxis und angesichts neuer Herausforderungen auswirken.

Zweitens können sich Institutionen und ihr Design ungeplant und von den Mitgliedstaaten ungewollt verändern: sei es durch eine Veränderung der Mitgliedschaft, sei es unter dem Einfluss nichtstaatlicher Akteure oder unter dem Einfluss ihrer ständigen Organe. Die Forschung schaut insbesondere auf die Organe internationaler Organisationen wie Se- kretariate, Schiedsgerichte oder operative Büros. Sie geht davon aus, dass Staaten Kompetenzen an derartige Organe delegieren und die damit einhergehenden Kontrollverluste in Kauf nehmen, weil sie auf mehr Effektivität hoffen. Allerdings können diese Organe ihre Spielräume in einer Weise nutzen, die den Interessen einzelner Mitglieder entgegenläuft. Auf diese Weise würde die Legitimation der Institution, die auf der Zustimmung aller Mitgliedstaaten ruht, gefährdet. ${ }^{3}$

Drittens erweisen sich Institutionen als starr. Wenn sich die Interessen eines oder mehrerer Teilnehmerstaaten verändern, bedeutet dies nicht, dass sich auch die Regeln und das Design der Institution wandeln. Diese Starrheit wird insbesondere dann zum Problem, wenn die Normen und Regeln einer Organisation eine anzustrebende Ordnung widerspiegeln, das bedeutet, wenn es darum geht, die Sozialisation (neuer) Mitgliedstaaten und ihre Annäherung an diese Ordnung abzusichern. Gelingt die Transformation nicht, öffnet sich eine Lücke zwischen den institutionellen Normen und der realen Ordnung. Eine solche Lücke tat sich in den letzten Jahren insbesondere im postkommunistischen Raum Eurasiens auf. ${ }^{4}$

Werden Institutionen einer Reihe von Mitgliedern fremd, reagieren diese Staaten in der Regel mit Strategien, die vom Austritt und dem Aufbau alternativer Institutionen über Widerstand von innen, die Kürzung von Ressourcen (wie Mitgliedsbeiträgen) bis hin zu Reformbemühungen reichen. ${ }^{5}$

Wird die Mitgliedschaft trotz zunehmender Heterogenität innerhalb der Institution beibehalten, entsteht ein grund- 
sätzliches Dilemma zwischen der Handlungsfähigkeit und der Legitimität der Institution. Dann gibt es zwei Reformpfade:

- Zum einen können Staaten versuchen, das Aufgabenspektrum der Organisation auf die Bereiche mit gemeinsamen Interessen zu beschränken. Sie können dann die Handlungsfähigkeit auf diesen Feldern stärken, indem sie weiterhin Kompetenzen an die Organe der Institution delegieren. Die Legitimität würde so zwar eingeschränkt. Aber durch die Beschränkung der Aufgaben auf die Felder gemeinsamer Interessen bleibt der Verlust begrenzt.

- Zum anderen können Staaten an der Breite der Aufgabenfelder festhalten. Sie würden dann aber die Spannung zwischen Legitimität und Effektivität auflösen, indem sie Abstriche bei der Handlungsfähigkeit machen.

\section{Wieso funktionierte die KSZE?}

Die KSZE entsprach im Wesentlichen der zweiten Variante. Ihr Ziel war die Kodifizierung der friedlichen Koexistenz zwischen den beiden Blöcken in Ost und West, die auf der allmählich gereiften Erkenntnis beruhte, dass ein Regimewechsel hier wie dort auf absehbare Zeit nicht zu erwarten war. Für die Sowjetunion und den Warschauer Pakt sowie insbesondere für Polen und die DDR ging es darum, dass der Westen den territorialen Status quo in den durch die kommunistischen Regime gesetzten Grenzen aner- kannte. Für die NATO und insbesondere die Bundesrepublik Deutschland war das Ziel, eben diese Grenzen durchlässiger zu machen (sowie trotz der Anerkennung des territorialen Status quo die deutsche Einheit nicht ein für alle Mal auszuschließen). Die KSZE beruhte folglich auf einem Kompromiss, der in antagonistischer Kooperation auf beiden Seiten widerwillig eingegangene Konzessionen für einen modus vivendi beinhaltete. Dieser Kompromiss wurde daher von Konservativen in Ost wie West prinzipiell abgelehnt.

Die extrem heterogenen Interessen und politischen Ordnungsvorstellungen blieben aber bestehen. Daher war mehr als die Schlussakte von Helsinki 1975 - gewissermaßen der Höhe- und Schlusspunkt der Entspannungsperiode zwischen Ost und West - nicht zu erreichen. Aufzulösen waren diese Differenzen nicht, und so stand im Helsinki-Dekalog die vom Osten gewünschte „Nichteinmischung in innere Angelegenheiten" unverbunden neben der vom Westen geforderten „Achtung der Menschenrechte und Grundfreiheiten, einschließlich der Gedanken-, Gewissens-, Religionsund Überzeugungsfreiheit". ${ }^{6}$ Dieser Widerspruch findet sich heute nahezu wortgleich wieder. Es war daher auch kein Wunder, dass die 1973 parallel eingeleiteten Mutual-and-Balanced-Force-Reductions-Verhandlungen in endlosen Streitereien versandeten. Und an die Schaffung einer beide Seiten verbindenden Organisation war überhaupt nicht zu denken: Bis 1989 fanden gerade einmal drei mehrjährige KSZE-Folgekonferenzen statt, in Belgrad (1977-1979), Ma- 
drid (1980-1983) und Wien (1986-1989). Von diesen verliefen insbesondere die ersten beiden weitgehend ergebnislos. Angesichts der neuerlichen Verschlechterung des Ost-West-Klimas Anfang der 1980erJahre - aufgrund der sowjetischen Afghanistaninvasion und des NATO-Doppelbeschlusses - war das kaum überraschend.

\section{Chancen und Grenzen der OSZE}

Dass am 1. Januar 1995 aus der lockeren „Konferenz" über Sicherheit und Zusammenarbeit eine echte „Organisation“ wurde, verdankt sich allein der Auflösung des Ostblocks. Bei den ehemaligen Mitgliedern des Warschauer Pakts entstand der Wunsch nach einer „Rückkehr nach Europa“, Russland strebte eine „Rückkehr zur Zivilisation" an, während bei den übrigen Nachfolgestaaten der UdSSR die Orientierung diffus blieb. Auf westlicher Seite änderte sich dagegen erst einmal nichts. Das hatte auch für die OSZE Konsequenzen, denn mit einer Organisation hatte sie in erster Linie den Namen gemein. Ihr rechtlicher Status blieb ebenso ungeklärt wie ihre Kompetenzen, trotz der Schaffung ständiger Organe und ihrer vergleichsweise guten personellen und materiellen Ausstattung. Bei ihrer eigentlichen Aufgabe, die Sicherheit in Europa zu organisieren, zehrte die OSZE von den Errungenschaften der Vergangenheit: dem Vertrag über Konventionelle Streitkräfte in Europa sowie den vertrauensund sicherheitsbildenden Maßnahmen des KSZE-Prozesses. So verkörperte die OSZE vor allem eine Vision: den Abschied vom „Erbe der Vergangenheit“ und den Aufbruch in „ein neues Zeitalter der Demokratie, des Friedens und der Einheit", wie es gleich zu Beginn der "Charta von Paris für ein Neues Europa“ heißt. ${ }^{7}$ Die Charta von Paris wurde 1990 verabschiedet, vom ersten KSZE-Gipfeltreffen nach Helsinki 1975. Von dieser Vision ist wenig geblieben. Vielmehr offenbarte die OSZE im Laufe der Zeit praktisch alle der oben skizzierten Grenzen internationaler Organisationen.

Für den Westen manifestierte sich die Vision vor allem im gemeinsamen Bekenntnis zu Demokratie und Marktwirtschaft. In der Praxis bedeutete dies, dass sich der einstmals real existierende Sozialismus nach westlichem Skript und unter tatkräftiger Mithilfe des Westens wandeln musste. Mit wachsendem Abstand von den Wirren des Systemumbruchs weckte dies in Russland erneut die Geister einer nachholenden Entwicklung, wie sie bereits seit dem 19. Jahrhundert bekannt sind. Für Russland manifestierte sich die Vision der OSZE im Unterschied zum Westen vor allem in der Schaffung einer gesamteuropäischen Sicherheitsordnung. Diese sollte ursprünglich im Zuge der deutschen Vereinigung unmittelbar an die Stelle der Militärblöcke treten. Das scheiterte vor allem am amerikanischen Wunsch, mit der NATO nicht nur einen festen Anker für das vereinigte Deutschland bereit $\mathrm{zu}$ halten, sondern mit ihr auch die US-Präsenz in Europa abzusichern. ${ }^{8}$ Nach dieser Niederlage modifizierte Russland seine Präferenzen: So forderte Moskau im Verlauf der 1990er Jahre, die OSZE an die Spitze der europäischen Sicherheitsordnung zu setzen, wovon Ende der 1990er Jahre lediglich noch 
die Idee blieb, dass die OSZE die bestehenden Organisationen wenigstens koordinierend ergänzen solle. So stand es im russischen Vorschlag für die „Charta der Europäischen Sicherheit". ${ }^{9}$ Am westlichen NATO-Zentrismus änderte all dies nichts - und damit auch nichts an der Außenseiterrolle, die Russland in dieser europäischen Ordnung spielte.

Ursprünglich war es folglich der Westen, der mit seinem Beharren auf der NATO die prospektive ordnungs- und sicherheitspolitische Einheit des Kontinents verhinderte. Mit der autokratischen Regression Russlands verlor diese Inkohärenz zwischen dem gesamteuropäischen ordnungspolitischen Anspruch und der teileuropäischen realpolitischen Praxis an Relevanz. Denn mit einem Russland, das sein Selbstverständnis nunmehr in prinzipieller Abgrenzung vom Westen formuliert, sind allenfalls noch vertragliche Abmachungen kooperativer Sicherheit möglich. Für solche vertragliche Abmachungen stellt die OSZE aus Sicht des Westens eine geeignete Plattform dar, etwa mit dem Forum für Sicherheitskooperation, dem Konfliktverhütungszentrum sowie dem Wiener Dokument über Vertrauensund Sicherheitspolitische Maßnahmen. Russlands Verhältnis zur OSZE ist dagegen ambivalenter. Denn die OSZE verkörpert einerseits die sicherheitspolitische Marginalisierung des Landes auf dem europäischen Kontinent, andererseits stellt sie die einzige institutionalisierte Plattform dar, auf der Moskau Stimme und Gewicht hat. Diese Ambivalenz zeigt sich in einer eigentümlichen Mischung aus aktiver Mitwirkung in den OSZE-Gremien und fundamentaler Kri- tik - bis hin zu Forderungen, die Organisation zu verlassen. Solche Forderungen sind in der Moskauer Expertengemeinde durchaus populär, verbunden mit der optimistischen Erwartung, dass die OSZE dann ohnehin kollabieren werde.

Die russische Kritik wird seit 2004 mit wachsender Intensität vorgetragen und offenbart nachgerade klassisch die Lücke, die sich zwischen den russischen Interessen und den einst konsensual vereinbarten Regeln und Verfahren der OSZE aufgetan hat. Die Kritik richtet sich vor allem gegen den geografischen Fokus der OSZE-Aktivitäten „östlich von Wien“, ihre Fokussierung auf die menschliche Dimension, etwa die im Osten sehr viel umfangreichere und kritischere Wahlbeobachtung, und gegen die Vernachlässigung des sicherheitspolitischen Dialogs. ${ }^{10}$ Moskau will daher die Aktivitäten insbesondere des Warschauer Büros für demokratische Institutionen und Menschenrechte, des Beauftragten für Medienfreiheit sowie des Hohen Kommissars für Nationale Minderheiten durch eine Reihe von Maßnahmen begrenzen, die zwischen den Regierungen ausgehandelt werden. Sie sollen in das OSZE-Sekretariat integriert werden, detaillierte Regeln erhalten sowie dem Konsensprinzip der Mitgliedstaaten unterworfen werden. Zusammengefasst fordert Moskau „that the OSCE should be responsive to requests from host governments, rather than pursuing its own agenda, which it perceived as a Western one“. ${ }^{11}$

Im Ergebnis laufen die russischen Vorstellungen auf einen grundlegenden Umbau der Arbeitsweise sowie auf eine Abkehr von der normativen Grundlage der 
OSZE hinaus. Dies ist im bestehenden Format der Organisation kaum zu erreichen. Im Unterschied zur KSZE, als zwei Blöcke verhandelten und die 13 neutralen Staaten als Vermittler fungierten, ist die Situation in der OSZE weitaus komplexer. Ihr gehören 57 Teilnehmerstaaten an, von denen die des Westens zwar die Mehrheit bilden (29 NATO-Mitglieder plus sechs weitere der EU), alle aber jeweils eigene Interessen und Präferenzen verfolgen. In dieser Gemengelage einen Konsens herzustellen, selbst in elementaren Fragen wie der Besetzung der wenigen Funktionen und der Verabschiedung des Haushalts, erweist sich immer wieder als zeitraubende Herkulesaufgabe. ${ }^{12}$

\section{Die Antwort des pluralen Friedens}

Die Vision des „liberalen Friedens“, die auf Transformation und Integration setzt, hat sich nicht erfüllt. Sie ist die Essenz der Charta von Paris aus dem Jahr 1990. Mit wachsendem Nachdruck verwahrt sich Russland dagegen, subaltern in die liberale politische Ordnung eingefügt sowie in der vom Westen dominierten Sicherheitsordnung marginalisiert zu werden. Es beharrt stattdessen darauf, gleichberechtigt sowie unter Anerkennung normativer Differenz zu kooperieren. Die anderen eurasischen Autokratien in der OSZE artikulieren dies im Windschatten Russlands weniger deutlich, verfolgen aber das gleiche Ziel. Statt dem proklamierten Ziel des „Europe, whole and free“ zu dienen, riss die wertebasierte Strategie folglich neue Gräben auf. Diese Strategie verwandelte sich in eine Ein- flusszonenpolitik mit wachsenden ideologischen Untertönen und führte für alle Beteiligten zu steigenden Kosten.

Der liberale Frieden hat sich in wachsende Widersprüche verwickelt und seine Erfolgsaussichten stehen sehr in Frage. Daher schlagen wir mit dem „pluralen Frieden" ein alternatives Leitbild vor, um die Beziehungen zwischen Russland und dem Westen zu organisieren. ${ }^{13}$ Es setzt darauf, Frieden durch Trennung (Dissoziation) zu erreichen. ${ }^{14}$ Dissoziation bedeutet dabei nicht, neue Mauern zu errichten. Vielmehr geht es um die klare Abgrenzung von Rechten und Pflichten. Der Grundgedanke ist einfach. Da der Anspruch, Frieden durch normative Verschmelzung unterschiedlicher Staaten zu erreichen, zu wachsenden Friktionen führt, schlagen wir vor, Frieden durch die Anerkennung normativer Differenz und Abgrenzung zu sichern - und dadurch der Kooperation eine neue und stabilere Grundlage zu verschaffen.

Dieses Leitbild des pluralen Friedens knüpt an die Erfahrungen der KSZE und der Ost-West-Entspannung an. Deren Logik bestand darin, die fundamentalen Unterschiede zwischen den Blöcken nicht länger durch Konfrontation überwinden zu wollen. Indem dem Kontrahenten die Existenzberechtigung abgesprochen wurde, war die Konfrontation über Jahrzehnte zementiert worden. Vielmehr galt es, die Unterschiede anzuerkennen, um sie nach und nach zu überwinden. Der plurale Frieden passt diese Erfahrungen den heutigen Verhältnissen an, die sich durch ein deutlich komplexeres Muster von Abgrenzung und Interdependenz auszeichnen. Beispielswei- 
se will er die Abgrenzung nicht wie bei der KSZE territorial vornehmen, sondern entlang funktionaler Bereiche. Das bedeutet, Russland zwar sicherheitspolitische Mitsprache einzuräumen, nicht aber in Bezug auf die wirtschaftlichen und politischen Verhältnisse in seinen Nachbarstaaten. Grundsätzlich gründet der plurale Frieden auf einer dissoziativen Strategie in jenen Bereichen, die sich als besonders konfliktträchtig erwiesen haben. Er will jedoch die gegenseitigen Abhängigkeiten nicht beseitigen, sondern gemeinsamen Regeln unterwerfen.

Der plurale Frieden redet also keineswegs einem Werterelativismus das Wort, wie er jenen Ansätzen nachgesagt wird, die universale Werte den nationalen Interessen unterordnen. Nicht die Werte stehen infrage, sondern deren Inanspruchnahme als Ziel und Mittel der internationalen Politik. Daraus folgen für einen möglichen Umbau der OSZE zwei gegensätzliche Strategien, die den Grad der Institutionalisierung erhöhen oder reduzieren.

\section{Zwei Pfade für den Umbau der OSZE}

Die OSZE trägt in ihrer gegenwärtigen Verfassung kaum dazu bei, die Spannungen zwischen Russland und dem Westen abzubauen. Vielmehr ist sie zu einer Bühne dieser Auseinandersetzung geworden. Der Westen beharrt in der Logik des liberalen Friedens auf dem institutionellen Status quo. Er hält daran fest, dass die in der Charta von Paris erstmals vereinbarten Normen weiterhin als zentrale Verhaltensmaxime dienen. Russ- land insistiert dagegen darauf, dass dieser Status quo die internationalen Vorstellungen und Kräfteverhältnisse nicht länger reflektiert. Es will die Aktivitäten der OSZE stärker seiner Kontrolle unterwerfen und seinen normativen Präferenzen mehr Gewicht verleihen oder diese zumindest nicht infrage stellen lassen. Das Ergebnis sind wechselseitige Blockaden und ein Durchwurschteln (muddling through $)^{15}$, das weder ein wirkungsvolles Konfliktmanagement noch gar eine Konflikttransformation erlaubt.

Insoweit besteht unter den professionellen Beobachtern der OSZE durchaus Konsens, es folgt jedoch - ganz in der Logik des „muddling through" - wenig mehr als die Forderung, der OSZE einen gewichtigeren Platz in der politischen Agenda ihrer Mitglieder einzuräumen ${ }^{16}$, ihr „substantiell mehr politische Aufmerksamkeit und Ressourcen“ zukommen zu lassen ${ }^{17}$ oder „eine Rückkehr zur Diplomatie“ einzuleiten. ${ }^{18}$ Dies sind indes nur Appelle. Sie basieren auf der pessimistischen Einschätzung, dass unter den aktuellen Umständen im besten Fall nur kleine Schritte möglich seien - mit dem Ziel einer „pragmatischen Kooperation“ oder eines neuen „Modus Vivendi“, da die Verständigung auf einen neuen Status quo voreilig sei. ${ }^{19}$

Das ist wohl so, entbindet aber nicht davon, die konzeptionellen Grundlagen der OSZE zu überprüfen. Denn die Voraussetzungen haben sich geändert: Es bestehen normative Unterschiede und eine Konfrontation, die sich immer mehr verschärft. Im Gegensatz zum klassischen Ost-West-Konflikt, als beide Blöcke vollständig gegeneinander abgeschirmt wa- 
ren, gibt es neben der politisch-militärischen Konfrontation heute indes auch Zusammenarbeit in vielen Bereichen. Das gilt vor allem für das gemeinsame ökonomische Interesse, aber auch in der menschlichen Dimension. Allerdings leidet diese Kooperation zusehends unter ihrer wechselseitigen "Securitization" und damit ihrer Wahrnehmung als Risiko für die eigene Sicherheit. So hatten Sanktionen und Gegensanktionen bereits beträchtliche Abkoppelungseffekte zur Folge, um vermeintliche oder tatsächliche Verwundbarkeiten zu reduzieren. Im Zuge dessen mutiert Interdependenz immer stärker zum einseitig wahrgenommenen Risiko. Der Minimalkonsens wäre folglich, diese Felder der Kooperation zu stabilisieren und gegen die Felder der Konfrontation abzuschirmen, damit sie nicht für politische Positionsgewinne instrumentalisiert werden.

Das bloße Beharren auf der normativen Grundlage der OSZE reicht hier nicht aus. Zwar bekennen sich alle sowohl zu den Helsinki-Prinzipien als auch zur Charta von Paris, jedoch mangelt es an der Umsetzung. Die Auseinandersetzung darüber lähmt die OSZE in ihrer bestehenden Verfassung. Denn Russland hält dem westlichen Beharren auf der universellen Geltung der liberalen Werte als raison d'être der OSZE und als Basis einer umfassenden Sicherheit mit wachsendem Nachdruck eigene ideologische Konstrukte entgegen. ${ }^{20}$

Wenn folglich trotz normativer Heterogenität an einer umfassenden Mitgliedschaft festgehalten werden soll - und das ist im Sinne der europäischen Sicherheit unverzichtbar -, stehen nur die beiden bereits benannten Pfade eines Umbaus der OSZE zur Verfügung: die Erhöhung des Grades der Institutionalisierung oder dessen Absenkung.

\section{Aufwertung der OSZE zu einem handlungsfähigen Instrument der Friedenssicherung und Konfliktbearbeitung}

Der erste Reformpfad führt zu einer OSZE, die auf die Dimension der militärpolitischen Sicherheit eingegrenzt und institutionell vertieft ist. Ein solcher Fokus markiert noch am ehesten einen Bereich paralleler Interessen, auf dem die Teilnehmerstaaten komparative Vorteile der OSZE und auch Handlungsbedarf sehen, um das (wachsende) Konfliktpotenzial einzuhegen. Die zentralen sicherheitspolitischen Aufgaben liegen auf dem Tisch:

- Zu ihnen gehört seit den 1990er-Jahren, einen Ausgleich zwischen den Prinzipien der souveränen Gleichheit und freien Bündniswahl einerseits und der ungeteilten Sicherheit andererseits zu finden sowie Mechanismen der Sicherheit für jene Staaten zu identifizieren, deren bündnispolitischer Status umstritten ist. ${ }^{21}$ Dies könnte im Sinne des pluralen Friedens nach funktionalen Kriterien geschehen, die sich am Konfliktgehalt und Kooperationspotenzial der Politikfelder Sicherheit, Wirtschaft und Herrschaft orientieren. ${ }^{22}$

- Darüber hinaus geht es in jüngerer Zeit darum, Regeln für die Grauzo- 
ne informationeller grenzüberschreitender Aktivitäten zu schaffen.

- Und schließlich betrifft dies den großen Bereich der konventionellen Rüstungskontrolle, der militärischen Vertrauensbildung und der Konfliktprävention bzw. des Konfliktmanagements in Europa - insbesondere in jenen Regionen, die nicht von den politisch-militärischen Integrationsprojekten des Kontinents erfasst sind.

Bei allen und insbesondere dem dritten sicherheitspolitischen Aufgabenfeld ist die OSZE auch in operativer Hinsicht gefragt. Um trotz der vielfältigen Konflikte zwischen ihren 57 Teilnehmerstaaten und der oben erwähnten Blockaden die Handlungsfähigkeit der OSZE in diesen Aufgabenfeldern zu sichern, wäre ihre institutionelle Stärkung nötig. Das verlangt zum einen den Ausbau von Organen, damit die OSZE effektiv im Bereich der Früherkennung, der Mediation und der Friedenssicherung agieren kann. Um diese Strukturen effektiv zu verwalten und zu führen, braucht es einen starken Generalsekretär mit einem breiten organisatorischen Unterbau. Vorbild sind die Organe der Vereinten Nationen im Bereich der Friedenssicherung. Damit die OSZE effektiv als regionale Sicherheitsorganisation im Sinne des Kapitels VIII der Charta der Vereinten Nationen handeln kann, bedarf es zudem Entscheidungsverfahren, die einen Ausgleich zwischen den Prinzipien der Legitimität und Effektivität ermöglichen. Vorschläge aus den 1990er-Jahren, die auf die Einrichtung eines europäischen „Executive Council“ ähnlich dem Sicherheitsrat der Vereinten
Nationen zielten, ${ }^{23}$ bieten hier Denkanstöße.

Voraussetzung für die Zustimmungsfähigkeit dieses Reformpfades ist freilich im Sinne des pluralen Friedens, sich auf Prinzipien der Koexistenz als völkerrechtliche Verpflichtungen zu einigen sowie den Aufgabenbereich der OSZE zu beschränken, um ihre Handlungsfähigkeit sicherzustellen.

\section{Rückbau der OSZE zu einer} konsensbildenden Konferenz

Der zweite Reformpfad zielt auf den Rückbau der OSZE zu einer Arena des Dialogs. Die OSZE würde an ihrer breiten Ausrichtung mit den drei Dimensionen festhalten. Statt aber die Einhaltung der liberalen Normen der Charta von Paris zur Richtschnur zu machen und ihre Organe darauf auszurichten, über die Umsetzung dieser Normen zu wachen, würde die OSZE als eine permanent tagende Konferenz operieren. Ein solches Arrangement bietet den Vorteil politischer Inklusivität und prozeduraler Informalität. Gerade weil sie auf effektive Verfahren verzichtet, um bindende Entscheidungen herbeizuführen, schafft sie - trotz normativer Unterschiede - Räume für Gespräche und die Suche nach Gemeinsamkeiten. Um Informalität zu gewährleisten, verzichtet dieses Arrangement auch auf institutionelle Zwangsmechanismen wie das indirekt wirkende „Naming and Shaming“. Semi-autonome Organe wie das Büro für demokratische Institutionen und Menschenrechte stellen aus dieser Sicht ein Hindernis dar, 
weil sie für einige Teilnehmerstaaten ein Risiko verkörpern und deren Bereitschaft zum Dialog schmälern. Im kritischen Bereich der menschlichen Dimension würde die OSZE zwar an ihren Normen festhalten, gleichzeitig aber politisch flexibel agieren. Dialog und stille Diplomatie können in vielen Fällen nicht nur prekäre Einzelfälle besser lösen, sondern sind auch geeignet, Regeln für den wechselseitigen Austausch zu vereinbaren.

Die Umwandlung zu einer dauerhaft tagenden Konferenz reduziert freilich die operative Handlungsfähigkeit der OSZE, denn als informelles Dach bleiben den beteiligten Staaten nur mehr Ad-hoc-Arrangements ähnlich der Special Monitoring Mission in der Ostukraine. Allerdings könnte die Bereitschaft dazu sogar wachsen, da die Teilnehmerstaaten nicht mehr fürchten müssen, dass ihnen durch permanente operative Arrangements die Kontrolle entgleitet.

\section{Trendumkehr mit und durch die OSZE?}

Trotz der hier genannten Reformmöglichkeiten sei vor Optimismus gewarnt. Die Krise der OSZE spiegelt den $\mathrm{Zu}$ stand der Beziehungen zwischen Russland und dem Westen. Entsprechend begrenzt sind die Chancen, den kausalen Zusammenhang umzudrehen und durch eine Reform und Reaktivierung der OSZE diese Beziehung, die für die Sicherheit in Europa zentral ist, zu entspannen. Die OSZE versammelt Staaten mit höchst unterschiedlichen Interessen, politischen Ordnungsvorstellungen und Erwartungen, die kaum unter dieses Dach passen. Zudem muss die OSZE in einem Feld, in dem verschiedene Organisationen konkurrieren, ihren komparativen Vorteil beweisen. Dies gelang ihr nach 1990 nur zum Teil und gelingt ihr seit Beginn der 2000er-Jahre immer weniger.

Dennoch bietet die OSZE Chancen, die aktuelle Hauptaufgabe zu bewältigen und die typischen "Steps to War" umzukehren. Diese Theorie über den Ausbruch von Kriegen, die auf Paul Senese und John Vasquez zurückgeht, nimmt an, dass territoriale Konflikte besonders gefährlich sind - gefährlicher als Regimekonflikte. Sie können insbesondere dann zum Krieg eskalieren, wenn die Akteure machtpolitische Strategien wie Machtdemonstrationen, Allianzbildung und Aufrüstung einsetzen und wenn dadurch Politikfeldgrenzen verschwimmen. ${ }^{24}$ Will man diesen Weg umkehren, kommt es darauf an, die Felder gemeinsamer Interessen zu stabilisieren, militärisches Vertrauen zu schaffen und die territorialen Konflikte zumindest einzufrieren. In Europa gilt dies mit besonderer Dringlichkeit für die Ostukraine.

Eine solche Umkehr wird die OSZE nicht allein schaffen können. Grundvoraussetzung ist die Einsicht, dass sich durch Druck und Drohungen von außen die andere Seite nicht verändern und nicht zum Nachgeben zwingen lässt. Diese Einsicht hat zwar in Moskau und in den westlichen Hauptstädten bislang nur wenige Anhänger, gewinnt aber mangels Alternativen an Gewicht. ${ }^{25}$ Die OSZE ist als Forum militärischer Vertrauensbildung und aufgrund ihres Engagements im Donbass in der Ukraine der richtige Ort, um einen solchen Weg aus der eska- 
lierenden Konfrontation zu organisieren. Allerdings ist ihr institutioneller Umbau, der normative Unterschiede in Rechnung stellt, die Voraussetzung dafür, dass sie wieder eine konstruktive Rolle spielen kann. Die hier vorgestellten Reformpfade sind Idealtypen und teilweise kombinierbar. Dennoch erscheinen sie als die am ehesten gangbaren Wege aus der Krise sowohl der OSZE als auch generell der Beziehungen zwischen Ost und West.

\section{Endnotes}

1 Wir danken vier anonymen Gutachtern für ihre wertvollen Hinweise und Anregungen, die wir im Rahmen unserer Möglichkeiten gerne aufgegriffen haben.

David A. Lake/Lisa L. Martin/Thomas Risse, „Challenges to the Liberal Order: Reflections on International Organization", in: International Organization 75:2, 2021, S. 225-257.

3 Michael Zürn, „Contested Global Governance", in: Global Policy 9:1, 2018, S. $138-145$.

4 Alexander Cooley, „Ordering Eurasia: The Rise and Decline of Liberal Internationalism in the Post-Communist Space", in: Security Studies 28:3, 2019, S. 588613.

5 Julia C. Morse/Robert Keohane, „Contested Multilateralism“, in: Review of International Organizations 9, 2014, S. 385-412.

6 KSZE, Schlussakte, Helsinki 1975, S. 6-8, www.osce.org/files/f/documents/6/e/3950 3.pdf

7 KSZE, Charta von Paris für ein Neues Europa, Paris 1990, S. 1, www.osce.org/fil es/f/documents/5/b/39518.pdf

8 OSCE Network of Think Tanks and Academic Institutions, The Road to the
Charter of Paris: Historical Narratives and Lessons for the OSCE Today, 2017, https://osce-network.net/file-OSCE-Netw ork/Publications/Road_to_the_Charter_ of_Paris_final_report.pdf

9 Wolfgang Zellner, „Russia and the OSCE: From High Hopes to Disillusionment", in: Cambridge Review of International Affairs, 18:3, 2005, S. 389-402.

10 Andrey Kortunov, „To Stay or Not to Stay? Seven Concerns Russia Has about the OSCE“, Russian International Affairs Council, 2021, https://russiancouncil.ru/e n/analytics-and-comments/analytics/to-st ay-or-not-to-stay-seven-concerns-russia-ha s-about-the-osce; Andrey Zagorsky, „The OSCE in Modern Europe", Russian International Affairs Council, 2015, http://russ iancouncil.ru/en/inner/?id_4=6865\#top-c ontent; Zellner, a.a.O. (Anm. 9).

11 Andrey Zagorsky, „Russia and the OSCE“, in: Frank Evers and Argyro Kartsonaki (eds.), The Future of the OSCE: Government Views, OSCE Insights 5/2021, Special Issue (Baden-Baden: Nomos, 2021), https://doi.org/10.5 771/9783748911456-05

12 Thomas Greminger, „Für eine effektivere OSZE: Praktische Empfehlungen eines ehemaligen Generalsekretärs", OSCE Insights 1/2021, (Baden-Baden: Nomos, 2022), https://doi.org/10.5771/978374891 1449-01

13 Matthias Dembinski/Hans-Joachim Spanger, „Pluraler Frieden“ - Leitgedanken $\mathrm{zu}$ einer neuen Russlandpolitik, HSFKReport Nr. 2, 2017, unter: https://www.h sfk.de/fileadmin/HSFK/hsfk_publikation en/report0217.pdf

14 Zur Dissoziation als Friedensstrategie siehe Werner Link, „Überlegungen zu einer strukturellen Konflikttheorie“, in: Gert Krell/Harald Müller (Hrsg.), Frieden und Konflikt in den Internationalen Beziehungen (Frankfurt: Campus, 1994), S. 99-115. 
15 Wolfgang Zellner, Using the OSCE more effectively - ideas and recommendations, CORE/IFSH, Paper for the Federal Foreign Office, 2020, https://ifsh.de/file/publ ication/2021_OSZE-Studie/20210301_US ING_THE_OSCE_MORE_EFFECTIVEL Y.pdf

16 OSCE Network of Think Tanks and Academic Institutions, Reviving Co-operative Security in Europe through the OSCE, 2015, https://osce-network.net/file admin/user_upload/publications/Revivin g_Co-operative_Security_in_Europe_thr ough_the_OSCE_web.pdf

17 Zellner, a.a.O. (Anm. 15).

18 OSCE, Panel of Eminent Persons, Back to Diplomacy: Final Report and Recommendations of the Panel of Eminent Persons on European Security as a Common Project, 2015, https://www.osce.org/files/ f/documents/2/5/205846.pdf

19 Zagorsky, a.a.O. (Anm. 11).

20 Dazu gehört wie im Kalten Krieg, liberale und soziale Rechte einander gegenüberzustellen, den liberalen Werten ihre universelle Geltung abzusprechen und mit der Kritik am westlichen Werteverfall eine ideologische Gegenoffensive zu starten. In der neuen Nationalen Sicher- heitsstrategie, die 2021 verabschiedet wurde, wendet sich Moskau erstmals ausführlich gegen eine „Verwestlichung“ als Sicherheitsbedrohung, um gleichzeitig die „traditionellen spirituell-moralischen Werte Russlands“ zu verteidigen (Ukaz Presidenta Rossijskoj Federacii, Strategija nacionalnoj bezopasnosti Rossijskoj Federacii, 2021, S. 34-38, http://scrf.gov.ru/ media/files/file/l4wGRPqJvETSkUTYmh epzRochb1j1jqh.pdf

21 Zagorsky, a.a.O. (Anm. 11); OSCE, Panel of Eminent Persons, a.a.O. (Anm.18), S. 14 .

22 Dembinski/Spanger, a.a.O. (Anm. 13), S. $29 \mathrm{ff}$.

23 Zellner, a.a.O. (Anm. 9), S. 392.

24 Jack S. Levy, „The ,paths-to-war concept", in: J. A. Vasquez (Eds.), What do we know about war? 2nd ed., Lanham, MD: Rowman and Littlefield, 2012, S. 281-290.

25 Walter Kemp/Reinhard Krumm (Eds.), Restoring European Security: From Managing Relations to Principled Cooperation, Friedrich-Ebert-Stiftung, Regional Office for Cooperation and Peace in Europe, 2021, http://library.fes.de/pdf-files/ bueros/wien/17733.pdf 